\title{
ASSESSMENT OF ADVANCED REACTOR CORE PROTECTION SYSTEM FOR PRESSURIZED WATER REACTOR
}

\author{
Wang-Kee In \\ KAERI, 1045 Daedeok-daero, \\ Yuseong, Daejeon, Rep. Korea \\ Phone : +82-42-868-2823, \\ Fax : $+82-42-863-0565$ \\ wkin@kaeri.re.kr
}

\author{
Young-Ho Park \\ Kepco Nuclear Fuel, Yuseong, \\ Daejeon, Rep. Korea \\ Phone : +82-42-868-1480 \\ yhpark@knfc.co.kr
}

\author{
Chang-Ho Kim \\ Kepco E\&C, Yuseong, \\ Daejeon, Rep. Korea \\ Phone : +82-42-868-4327 \\ kimch@kepco-enc.com
}

\author{
Seung-Yeob Baeg \\ Doosan Heavy Industries, Yongin-si, Rep. Korea \\ Phone : +82-31-270-7040 \\ Seungyeob.baeg@doosan.com
}

\author{
Tae-Young Yoon \\ Kepco Nuclear Fuel, Daejeon, Rep. Korea \\ Phone : +82-42-868-1489 \\ tyyoon@knfc.co.kr
}

Keywords: Core protection, RCOPS, CPCS, Functional algorithm, Software, DNBR, LPD.

\begin{abstract}
An advanced core protection system for a pressurized water reactor (PWR), RCOPS, was developed by adopting a high performance hardware platform and optimal system configuration. The functional algorithms of the core protection system were also improved to enhance the plant availability by reducing unnecessary reactor trips and increasing operational margin.

The RCOPS used a safety class programmable logic controller POSAFE-Q PLC that was developed as a controller for the nuclear reactor safety system. It consists of four independent safety channels providing a two-out-of-four trip logic. The reliability analysis using the reliability block diagram method showed the unavailability of the RCOPS to be lower than the conventional system. The failure mode and effects analysis (FMEA) demonstrated that the RCOPS does not lose its intended safety functions for most failures. New algorithms for the RCOPS functional design were implemented in order to avoid unnecessary reactor trips by providing auxiliary pre-trip alarms and signal validation logic for the control rod position. The new algorithms in the RCOPS were verified by comparing the RCOPS calculations with reference results. The new thermal margin algorithm for the RCOPS was expected to increase the operational margin to the limit for departure from nucleate boiling ratio (DNBR) by approximately $2 \%$.
\end{abstract}

\section{INTRODUCTION}

Most of the existing PWR plants employ an either analog protection system or a digital protection system to provide core protection against fuel centerline melting and departure from nucleate boiling. The analog PWR plants including six units in Korea adopted the thermal overpower and overtemperature $\Delta \mathrm{T}(\mathrm{OP} \Delta \mathrm{T}$ and $\mathrm{OT} \Delta \mathrm{T})$ protection system [1]. The overpower $\Delta \mathrm{T}$ trip $(\mathrm{OP} \Delta \mathrm{T})$ provides protection against exceeding fuel rod design limits for accidents involving overpower excursions. For normal operation, the temperature rise through the reactor vessel $(\Delta T)$ is approximately proportional to the power. There is, however, a slight dependence on pressure and reactor inlet temperature due to the change in the density and heat capacity of the water. The overtemperature $\Delta \mathrm{T}$ trip (OT $\Delta \mathrm{T}$ ) protects the core against departure from nucleate boiling for any combination of power, pressure, temperature, and axial core power distributions.

The digital PWR plants used the digital computer based portion of the reactor protection system which is called the Core Protection Calculator System (CPCS). The CPCS [2] provides two of the reactor protection system functions by calculating real-time values of DNBR and Local Power Density (LPD) based on live sensor signals used to synthesize a core axial power distribution and to compute appropriate thermal-hydraulic parameters. These calculated values of DNBR and LPD are continuously compared to plant specific trip limits. The CPCS has been employed in eight PWR units (OPR1000) in Korea in addition to the seven PWR units in USA.

An advanced core protection system, RCOPS (Reactor Core Protection System), has been developed for the localization of the CPCS for the digital PWR plants in Korea $[3,4]$. The newly designed RCOPS is a digital core protection system which provides core protection functions based on two reactor core operation parameters, DNBR and LPD. It generates a reactor trip signal when the core 
condition exceeds the DNBR or LPD design limit. The RCOPS adopted the safety system programmable logic controller (PLC), POSAFE-Q PLC with a software development tool to implement custom function blocks. The functional algorithms in the RCOPS were also improved to enhance the plant availability by reducing unnecessary reactor trips and increasing operational margin

This paper describes the system design and algorithm improvements of the RCOPS. The system verification and validation for the RCOPS were performed by testing the algorithm and software. New algorithms in the RCOPS were verified by comparisons with the expected results. The system analysis was conducted to estimate the unavailability and failure mode effects of the RCOPS. The thermal margin assessment for the RCOPS was also made to predict the change in the DNBR margin.

\section{RCOPS DESCRIPTIONS}

The RCOPS provides four independent channels of DNBR and LPD trip signals to the reactor protection system. Each channel consists of a Core Protection Processor (COPP) which is an independent computer with a complete complement of independent sensors for ex-core neutron flux at three levels, core inlet temperature, core pressure, control rod position, etc. In addition, the RCOPS includes four Control Element Assembly Processors (CEAPs) which evaluate the impact of single CEA misalignments and pass appropriate adjustment factors to the COPP channels. Two channel communication processors (CCP1 \& CCP2) in each channel are used to share the CEA position signals from two reed switch position transmitters, RSPT1 and RSPT2. Figure 1 shows the system configuration of the RCOPS.

The initial digital core protection system CPCS was developed in the 1970's and has being operated in numerous PWR plants for more than two decades. The operational experience with the CPCS requires an improvement in the algorithm in order to increase the plant availability. Hence, new algorithms for the RCOPS development were selected to reduce unnecessary reactor trips and increase the DNBR margin. The new algorithm features for the RCOPS are the improvement of DNBR calculation algorithm, the addition of pre-trip alarm generation for auxiliary trip function, the improvement of CEA signal checking algorithm and the actuation of reactor power cutback system for a 12-finger CEA drop.

The minimum DNBR (MDNBR) in the CPCS is calculated using a simplified thermal-hydraulic program, CETOP [5] which was developed to quickly calculate the MDNBR in a PWR core. It calculates the minimum DNBR based on a four-channel core model. A three-dimensional transport coefficient model is introduced to radially group the flow subchannel into four channels for the thermal-hydraulic calculation. The CETOP program solves the transport coefficient conservation equations by the finite-difference method. The conservation equations in the finite-difference form are solved by a non-iterative numerical scheme, i.e., a prediction-correction scheme. The prediction-correction method is a non-iterative numerical scheme which provides a fast solution for thermal-hydraulic parameters at each axial elevation from the core inlet to outlet.

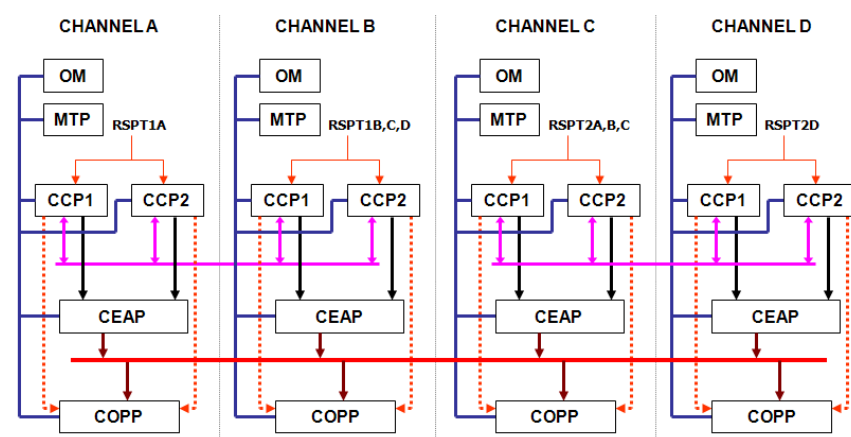

Figure 1. SYSTEM CONFIGURATION OF THE RCOPS.

There are two versions of the CETOP program available depending on its application, i.e., CETOP-D and CETOP2. The CETOP-D [6] is a reference faster-running DNBR code for use in the design calculations and safety analysis. The CETOP2 program is the simplified version of the CETOP-D code for the on-line calculation of MDNBR in the CPCS. The CETOP2 program uses an optimum value of enthalpy transport coefficient which is determined by a tuning process for the reference faster-running DNBR code, CETOP-D $a$ priori. In order to remove the DNBR calculation uncertainty in the CETOP2 due to its model simplification and eliminate the CETOP2-to-CETOP-D tuning process, the CETOP2 program in the CPCS was replaced with the CETOP-D code in the RCOPS.

The CETOP-D code calculates the MDNBR in a hot assembly of the core using the four flow channel model in Fig. 2. One-fourth (1/4) of the hot assembly and the remainder of the core are modeled as two individually lumped subchannels, i.e. channels 1 and 2. The hot assembly is modeled by three lumped channels, i.e. channels 2, 3, and 4. The hot subchannel is channel 4 . Channel 2 is a quadrant of the hottest assembly in the core and Channel 1 is an assembly representing the average coolant conditions for the remaining portion of the core. Lumped channel 2 includes channels 3 and 4. Channel 3 lumps the subchannels adjacent to the MDNBR hot channel 4. The radial rod peaking factors and the inlet mass flux of the hot assembly are selected from a detailed subchannel analysis so that under these two conditions the MDNBR will be calculated conservatively as compared to a detailed assembly-by-assembly analysis. This detailed analysis adopts different radial rod peaking factor distributions and mass fluxes for different assemblies. The governing equations and non-iterative numerical scheme used in the CETOP-D code are described in detail in the previous study [7].

There is a pre-trip alarm function for the main CPCS trip functions (DNBR and LPD trips). However, there is no pre-trip alarm function for auxiliary trips such as VOPT (Variable Overpower Trip), ASGT (Asymmetric Steam Generator Trip) and range trips. Among the above auxiliary trip functions, Axial Shape Index (ASI) range trip and ASGT trip were selected to add a pre-trip alarm function for the RCOPS. If a compensated cold leg temperature difference is greater than the pre-trip setpoint, or the hot pin ASI value is 


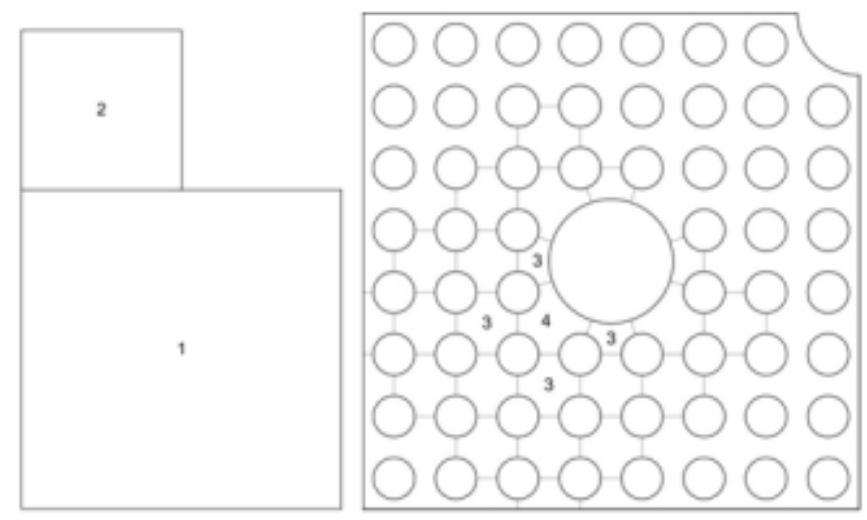

Figure 2. SCHEMATIC OF FOUR FLOW CHANNEL MODEL.

not within the auxiliary pre-trip setpoints, the auxiliary pre-trip alarm flag will be set and the pre-trip alarm will be initiated. It is expected that the pre-trip alarm for auxiliary trips will prevent the reactor trip by an appropriate operator action.

The Korean optimized power reactors (OPR1000) have experienced several reactor trips due to a false CEA position signal. Because there is a CEA position latching problem due to the different upward rate-of-change constant (TFU) from the downward one (TFD), some consecutive CEA noise signal could cause a wrong CEA position. In case of a 12-finger CEA position, a CEA deviation penalty factor results in a reactor trip. Therefore it is necessary to enhance the CEA signal checking algorithm. Based on the basic concept of the CEA free drop, i.e., consecutive CEA drop signals for real CEA dropping, an additional CEA free drop checking algorithm was added in the CEAP program. In order to avoid the CEA position latching problem due to the different upward and downward rate-of-change constants, the normal rate-of-change filter is introduced and the two consecutive dropping signals are only considered as free drop.

The OPR1000 plants have experienced several reactor trips due to a 12 -finger CEA drop. The basic concepts to prevent reactor trip for a 12 -finger CEA drop are to delay applying the penalty factor and to reduce the core power by the actuation of reactor power cutback system (RPCS). Therefore, the RCOPS algorithms were modified to initiate RPCS operation and not to apply penalty factors for short periods. If there is a 12-finger CEA drop and RPCS is not actuating already, RPCS actuation signal will be set. Then, RPCS will be actuated to reduce core power. In this case additional RPCS flag with a 12-finger CEA drop will be set instead of original RPC flag. This advanced algorithm will not generate reactor trip for an actual or noise 12 -finger CEA drop signal.

\section{SYSTEM VERIFICATION AND VALIDATION 3.1 Algorithm Verification}

The new algorithm for the RCOPS DNBR calculation was verified by comparing the RCOPS MDNBR with the CETOP-D MDNBR because the RCOPS DNBR algorithm

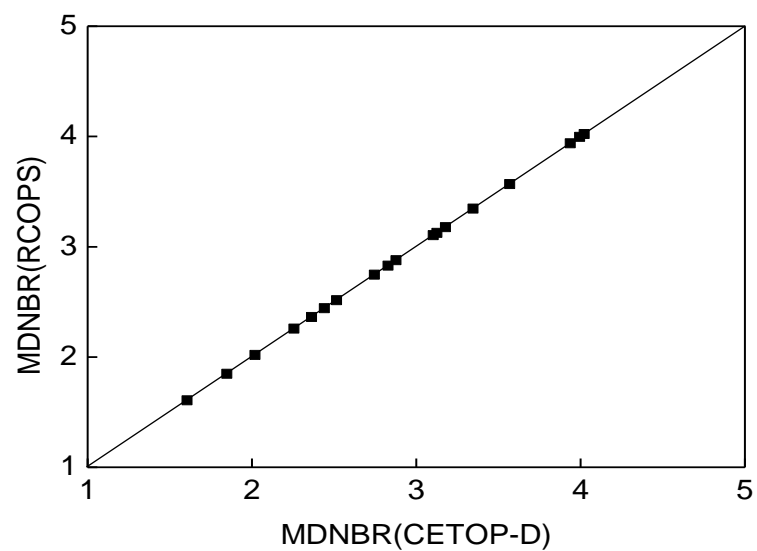

Figure 3. MDNBR COMPARISON FOR UNIFORM AXIAL POWER PROFILE.

was developed from the CETOP-D code. Figure 3 shows the MDNBR comparison for various core operating conditions using a uniform core axial power profile. It can be seen that the RCOPS MDNBR agrees well with the CETOP-D MDNBR. Figure 4 compares the RCOPS MDNBR error with respect to the CETOP-D MDNBR for a wide range of core operating conditions at three times in the cycle of a OPR 1000. Table 1 lists the range of core operating conditions used in this study. The operating conditions were randomly selected from the wide range of each variable assuming a uniform distribution. The RCOPS MDNBR error is defined as:

$$
E_{D N B R}=\frac{M D N B R(R C O P S)-M D N B R(C E T O P-D)}{M D N B R(C E T O P-D)}
$$

The RCOPS MDNBR error in Fig. 4 shows the positive value of mean error $(\mu)$ indicating that the RCOPS MDNBR is higher than the CETOP-D MDNBR. The MDNBR error at the beginning of cycle (BOC) is smaller than the ones at the middle of cycle (MOC) and the end of cycle (EOC). The MDNBR error is mostly due to the discrepancy in the core axial power profiles which were interpolated from given profiles in slightly different way in the RCOPS and CETOP-D. The RCOPS MDNBR error appears to be bounded by $1.5 \%$ which is small enough to verify the RCOPS DNBR algorithm.

A test simulation was conducted to verify the algorithm for the ASGT and ASI pre-trip alarms. The pre-trip alarm flag was set as designed when either the compensated cold leg temperature difference or the hot pin ASI violates its pre-trip limits. A noise signal for the CEA position was generated to simulate the CEA position latching problem. The new RCOPS algorithm for the CEA signal validation was found to select a correct signal as desired. Furthermore, the RCOPS algorithm was also found to not generate a reactor trip for an actual 12-finger CEA drop signal or for 12-finger CEA noise signals. The RCOPS will initiate the RPCS actuation signal upon the single 12-finger CEA deviation greater than the dead band. 
Table 1. REACTOR CORE OPERATING CONDITIONS.

\begin{tabular}{|l|c|c|}
\hline \multicolumn{1}{|c|}{ Core Parameter } & Unit & Value \\
\hline Inlet temperature & ${ }^{\circ} \mathrm{C}$ & $246-299$ \\
\hline Pressure & bar & $123-167$ \\
\hline Mass flux & $\%$ rated & $90-120$ \\
\hline Axial shape index & - & $-0.6-+0.6$ \\
\hline
\end{tabular}

\subsection{Software Verification and Validation}

The verification and validation of the RCOPS software were conducted by performing a module test and a unit test. The module test for a functional block is to compare the module result against the expected result by using pre-defined input values. A black box test was performed to verify all of the calculation branches by using a large number of test cases for each individual module. The RCOPS module tests were successful because the difference between the actual results and the expected results are within the acceptance criteria.

The objectives of the unit test are to verify that the RCOPS software has been properly integrated with the RCOPS system hardware and to confirm that the static and dynamic operation of the integrated system is consistent with that predicted by design analyses. These objectives are achieved by comparing the response predicted by the RCOPS off-line simulator or by hand calculations. The comparison is performed for a selected set of simulated input conditions. The unit test consists of a input sweep test, a dynamic software verification test (DSVT), and a live input single parameter (LISP) test. These tests are performed on the RCOPS single channel test facility using input/output simulator with the test software.

A preliminary input sweep test was performed to determine the processing uncertainties of the RCOPS algorithms as used in the system hardware that are inherent in the RCOPS designs. Approximately 2000 and 1200 test cases for COPP and CEAP software were selected to include different combinations of process inputs for the design qualification testing. These test cases cover the entire operating space of COPP and CEAP. The COPP input sweep test showed processing uncertainties of \pm 0.015 DNBR unit and $\pm 0.25 \%$ of core average linear heat rate, respectively. The CEAP input sweep test showed that the CEA penalty factor agrees with each other within its difference threshold, i.e., $5.0 \mathrm{E}-4$.

The DSVT is performed to verify that the dynamic response of the integrated RCOPS software is consistent with that predicted by design analyses. Ten(10) test cases were selected for the preliminary RCOPS DSVT. The initial values of DNBR and LPD calculated by the off-line simulator were found to agree with those generated by the on-line software within the processing uncertainties. The reactor trip time by the on-line software was also confirmed to be within allowable range which was determined by the off-line simulator with various time offsets.

The LISP test is used to verify the on-line software by the dynamic testing of the process input parameters and the
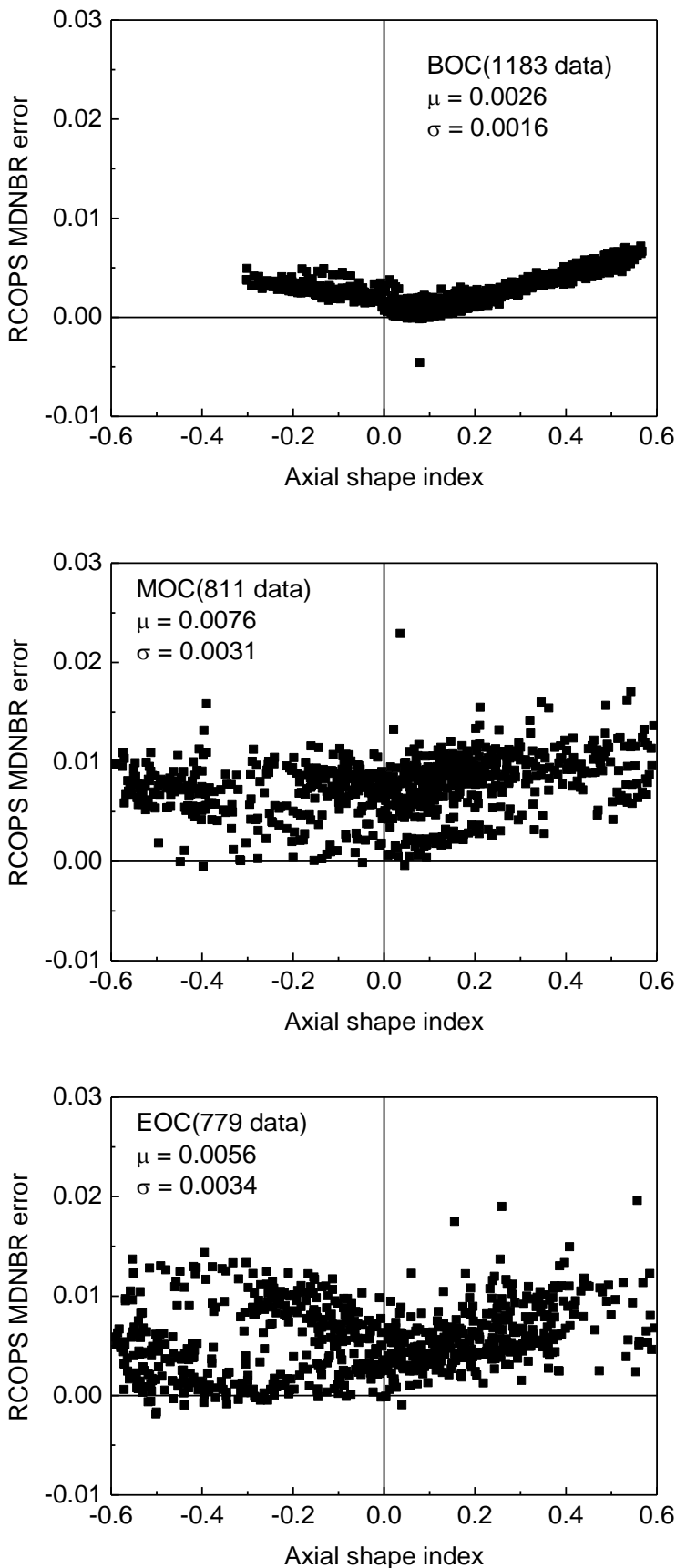

Figure 4. RCOPS MDNBR ERRORS AT THE BOC, MOC AND EOC.

related algorithms. The LISP test comprises five test cases: reactor coolant pump speed ramp, ex-core detector ramp, cold leg temperature ramp, primary pressure ramp, and CEA withdrawal. The minimum and maximum trip times of the on-line software were compared with those of the off-line simulator which includes the time offsets due to process signal noise, processor uncertainty, and program execution time. Three test cases were found to slightly fail the acceptance criteria due to the use of the preliminary time offsets associated with the RCOPS hardware design. It is therefore judged that the LISP test did not indicate any errors in the RCOPS software. 


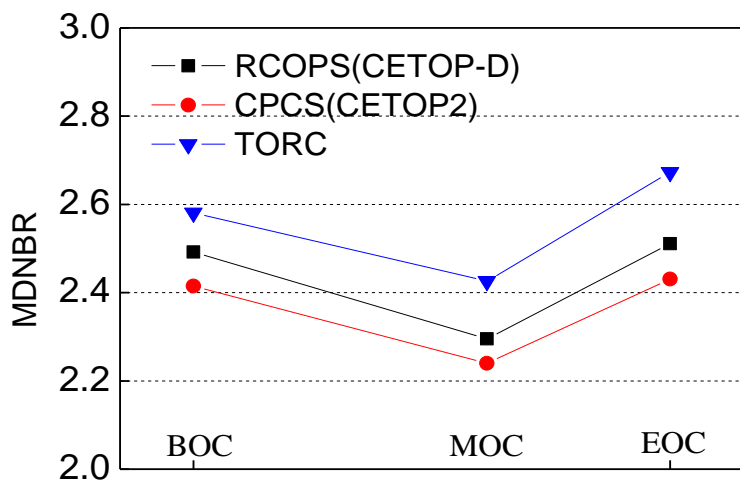

Burnup

Figure 5. MDNBR COMPARISON AT A NOMINAL OPERATING CONDITION OF OPR1000.

\section{SYSTEM PERFORMANCE ASSESSMENT 4.1 Thermal Margin Assessment}

The MDNBR values were calculated using the CPCS and RCOPS DNBR algorithms at a nominal operating condition of the OPR1000. Figure 5 compares the MDNBR values predicted at each time in the cycle, e.g., BOC, MOC and EOC. The CPCS and RCOPS MDNBR values were corrected by the DNBR uncertainty [8] which was determined based on the MDNBR by a reference subchannel code TORC [9]. The one-sided upper tolerance limits for the DNBR uncertainty distribution were applied to correct the CPCS and RCOPS MDNBR values. Since the CETOP-D program in the RCOPS is tuned to TORC in order to ensure a conservatism at a limiting condition, the RCOPS case predicted a lower nominal MDNBR than the TORC by approximately $5 \%$. The DNBR calculation algorithm in the RCOPS was considered to improve the DNBR margin by more than $2 \%$ with respect to the CPCS.

\subsection{Reliability and Failure Mode Analysis}

The unavailability of RCOPS is improved with respect to the CPCS by adopting a simplified system configuration. From the viewpoint of redundancy, the CPCS has a sensor redundancy feature such as preferred and alternate sources for target CEA positions, e.g., RSPT1A and RSPT2A in case of channel A. However the RCOPS has only I/O redundancy whereas only RSPT1A is measured by channel communication processors, CCP1 and CCP2. Both CPCS and RCOPS systems have their own advantages and disadvantages due to the different system configurations.

A reliability block diagram (RBD) method was used to calculate the reliability of the RCOPS and the CPCS in order to compare the reliabilities and characteristics of both systems on the same calculation basis. Isograph reliability software was used as a calculation tool for reliability analysis of both systems. Both systems were conservatively analyzed. The unavailability of RCOPS is $1.272 \times 10^{-4}$ failures per demand. This result is approximately $4 \%$ lower than the $1.328 \times 10^{-4}$ failures per demand for the CPCS.

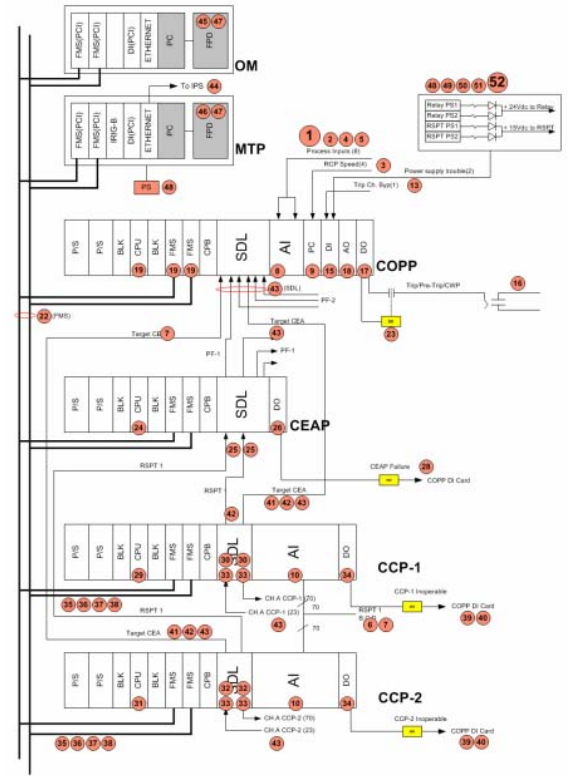

Figure 6. RCOPS FMEA BLOCK DIAGRAM.

The failure mode and effects analysis (FMEA) is the qualitative method to analyze the effects of single mode failures. FMEA was performed to assure safety and reliability of the RCOPS at the end of the design phase. More than 50 single mode failures for the RCOPS were defined on the block diagram. Figure 6 shows the block diagram for the RCOPS marked by unique numbers for each single mode failure. It was certain that the RCOPS does not lose its intended safety functions for most single mode failures. In the event of processor failures, the RCOPS will not have enough features to satisfy the system requirement. Hence a dry contact module was supplemented to generate channel trips using a watch dog timer.

\section{CONCLUSIONS}

A reactor core protection system, RCOPS, was developed by adopting a high performance hardware platform and new functional algorithms. The verification and validation for the RCOPS were performed by testing the algorithm and system software. The RCOPS MDNBR error is bounded by $1.5 \%$ which is small enough to verify the RCOPS DNBR algorithm. The new algorithms for pre-trip alarms and CEA signal validation were also verified against the expected results. Even if the LISP test showed some failures due to the preliminary design values of the RCOPS hardware, the module test and unit test did not indicate any errors in the RCOPS software.

Thermal margin and unavailability of the RCOPS were also estimated to assess the system performance over the conventional system, CPCS. The new DNBR algorithm in the RCOPS is expected to increase the DNBR margin by more than $2 \%$ with respect to the CPCS. The unavailability of the RCOPS was estimated to be $4 \%$ lower than the CPCS.

\section{ACKNOWLEDGEMENT}

The authors express their appreciation to the Korea Hydraulic and Nuclear Power and KEPCO Nuclear Fuel for 
financial support.

\section{REFERENCES}

[1] Han, K. I., 1984, J. KNS, Vol. 16(2), pp 106-115.

[2] Combustion Engineering Inc., 1974, CENPD-170.

[3] Baeg, S. Y., et al., 2007, European Nuclear Conference, September, Brussels, Belgium.

[4] Lee, S. H., et al., 2008, 16th Pacific Basic Nuclear Conference, October 13-18, Aomori, Japan.
[5] Chiu, C., 1981, Nuclear Engineering and Design, Vol. 64, pp 103-115.

[6] Combustion Engineering Inc., 1982, CEN-214(A)-NP.

[7] In, W. K., et al., 2009, Int'l Conf. on Nuclear Engineering (ICONE17), July 12-16, Brussels, Belgium

[8] In, W. K., et al., 2003, Korea Nuclear Society Spring Meeting, May 27-28, Kyoungju, Korea.

[9] Combustion Engineering Inc., 1977, CENPD-206-P. 